\title{
Pregnancy in systemic lupus erythematosus
}

\author{
C C Mok, R W S Wong
}

\begin{abstract}
Systemic lupus erythematosus (SLE) is an autoimmune disease that predominantly affects women of reproductive age. Pregnancy and its outcome is a major concern to most SLE patients. Queries regarding the risk of disease flares during pregnancy, chance of fetal loss, and the safety of various drugs are often raised. With the improvement in the understanding of the pathogenesis of SLE and the judicious use of immunosuppressive drugs, better disease control can now be achieved and SLE patients should not be deprived of the opportunity for bearing children. Prepregnancy counselling and close collaboration with other specialists such as the obstetricians and the perinatologists is essential in optimising the maternal and fetal outcome in lupus pregnancies. In this review, important issues regarding the fertility rate, optimal timing of conception, risk of disease flares during lupus pregnancy, pregnancy course, fetal outcome, safety of various drugs used for disease control during pregnancy and lactation, and contraceptive advice are discussed.

(Postgrad Med f 2001;77:157-165)
\end{abstract}

Keywords: systemic lupus erythematosus; pregnancy; flare; hormone

With the improvement in the survival and prognosis of systemic lupus erythematosus (SLE) in the recent decades, a significant proportion of SLE patients can now be maintained in disease remission. Uncertainties about the risk of childbearing to both the mother and the fetus have become a major worry from couples during clinic consultations. Advising couples on various aspects of pregnancy and contraception is always a difficult task. In this review article, common issues related to pregnancy in women with SLE are discussed. Appropriate individual based advice should be given to couples during prepregnancy counselling.

Fertility rate of SLE patients

Fertility of patients with SLE is usually unaltered by the disease. ${ }^{1}$ A pregnancy rate of
2.0 to 2.4 pregnancies per patient has been described, not only during disease remission but also during periods of disease activity. ${ }^{23}$ However, certain factors may contribute to a lower fertility rate in some patients. Menstrual irregularities with anovulatory cycles may occur during episodes of active disease and high dose corticosteroid treatment. ${ }^{4}$ Moreover, end stage renal failure secondary to lupus nephritis may result in amenorrhoea. Ovarian failure caused by cyclophosphamide treatment is another important factor for reduced fertility. ${ }^{5}$ The incidence of permanent amenorrhoea after cyclophosphamide treatment ranges from $11 \%$ to $59 \%$ and is dependent on the age of the patient and the cumulative dose of cyclophosphamide administered ${ }^{6}{ }^{7}$ In general, the risk of ovarian failure is much higher in patients who are treated by oral cyclophosphamide than intermittent intravenous pulse cyclophosphamide. In the absence of renal failure, cyclophosphamide treatment, active disease, or treatment with high dose steroid, patients with SLE should be as fertile as the general population and should not be discouraged from being pregnant, although they should be informed of the increased risk of pregnancy complications and fetal wastage. Appropriate individual based contraceptive advice should also be given for those who do not want to become pregnant because of social reasons.

\section{Pregnancy and flares of SLE}

Whether there is an increased risk of lupus flares during pregnancy when compared with those who are not pregnant is a subject of controversy. Numerous retrospective and uncontrolled studies conducted since the 1960s have reported exacerbation of SLE during pregnancy. Well designed prospective studies with controlled groups included for comparison are recently available but have yielded contradictory results (table 1) ${ }^{8-13}$ Lockshin et al, ${ }^{8}$ Mintz et $a l,{ }^{9}$ and Urowitz et $a l^{10}$ did not find any increased incidence of flares during pregnancy when compared with controls, while Wong et $a l,{ }^{11}$ Petri et $a l,{ }^{12}$ and Ruiz-Irastorza et $a l^{13}$ reported that pregnancy caused more lupus flares. The cause for the discrepancy of results from these studies is multifactorial and probably related to the differences in the definition

Table 1 Summary of six prospective controlled studies on lupus flares during pregnancy

\begin{tabular}{|c|c|c|c|c|c|c|}
\hline Authors & $\begin{array}{l}\text { Patients } \\
\text { (n) }\end{array}$ & $\begin{array}{l}\text { Pregnancies } \\
(n)\end{array}$ & Controls & $\begin{array}{l}\text { Use of disease } \\
\text { activity scale } \\
\text { (yes/no) }\end{array}$ & $\begin{array}{l}\text { Quantified } \\
\text { measure of flares } \\
\text { (yes/no) }\end{array}$ & Results \\
\hline Lockshin et $a l^{8}$ (1984) & 28 & 33 & Matched & Yes & No & No differences in flares \\
\hline Mintz et al ${ }^{9}(1986)$ & 75 & 102 & Unmatched & No & No & No differences in flares \\
\hline Urowitz et $a l^{10}$ (1993) & 46 & 79 & Matched & Yes & Yes & No differences in flares \\
\hline Wong et al ${ }^{11}$ (1991) & 22 & 29 & Unmatched & No & No & Increased flares in pregnancy \\
\hline Petri et al ${ }^{12}(1991)$ & 37 & 40 & $\begin{array}{l}\text { Unmatched } \\
\text { Post-delivery course }\end{array}$ & Yes & Yes & $\begin{array}{l}\text { Increased flares in pregnancy than controls } \\
\text { and after delivery }\end{array}$ \\
\hline Ruiz-Irastorza et al ${ }^{13}$ (1996) & 68 & 78 & $\begin{array}{l}\text { Matched } \\
\text { Post-delivery course }\end{array}$ & Yes & Yes & $\begin{array}{l}\text { Increased flares in pregnancy than controls } \\
\text { and after delivery }\end{array}$ \\
\hline
\end{tabular}


of lupus flares, assessment of disease activity, selection of the control group, and whether they matched well the disease characteristics of the pregnant patients, the proportion of patients with positive antiphospholipid antibodies (aPL) and abortion during early pregnancy who were excluded from analysis, and therapeutic strategies such as prophylactic steroids in late pregnancy. Certain physiological changes that occur during pregnancy may be misinterpreted as flares-for example, palmar erythema, transient facial blush, increase in proteinuria due to an increase in glomerular filtration rate, and postpartum alopecia. This may lead to an overestimation of the frequency of lupus flares during pregnancy. For this reason, modification of the commonly used disease activity indices has been proposed for the assessment of lupus activity during pregnancy. ${ }^{14}$

Despite the inconsistency of results generated, a common agreement from these studies is that lupus flares during pregnancy are fairly common, with a frequency of more than $57 \%$ and flare rates ranging from 0.06 to 0.136 per patient month. Every SLE patient should therefore be followed up during pregnancy under the assumption that there is a risk of flare.

Prediction of when an individual SLE patient will flare during the pregnancy course is difficult. Data regarding the frequency of flares during each trimester and the puerperium generated from five of the prospective studies described above are heterogeneous. ${ }^{9-13}$ Some studies reported that most flares occurred during the second trimester ${ }^{12}{ }^{13}$ but others described most flares at the first trimester. ${ }^{9} 10$ Wong et al reported an equal frequency of flares during the second and the third trimester $(38.5 \%)$ but no patients flared in the postpartum period. ${ }^{11}$ Prophylactic steroid treatment in late pregnancy to prevent postpartum flares, difference in proportion of patients with abortion or premature delivery, and hence difference in proportion of patients reaching the third trimester, and treatment given during the first trimester may well modify the course of SLE during pregnancy and probably contribute to the heterogeneity of the results from different studies. Nevertheless, it should be noted that SLE may flare at any trimester of pregnancy and in the postpartum period.

Lupus flares during pregnancy tend to be mild, with predominant arthritis and cutaneous manifestations. ${ }^{1391213}$ Some patients may experience minor flares with fever, fatigue, serositis, and thrombocytopenia. Exacerbation of SLE with major organ involvement such as the kidneys and the central nervous system, on the other hand, may occur up to $46 \%$ and $5 \%$, respectively, of patients. ${ }^{1112}$ Wong et al described six renal flares among 13 relapses $(46 \%)$ in 29 pregnancies of his cohort of SLE patients. ${ }^{11}$ Petri et al reported renal involvement in $43 \%$ of all flares during pregnancy, which was higher than those of the controls $(22 \%) .^{12}$ However, only $11 \%$ of all flares were regarded as severe flares according to the physican's global assessment. Ruiz-Irastorza et al also described that $23 \%$ of flares during pregnancies

\section{Box 1: Pregnancy and flares of SLE}

- Whether flares of SLE are more frequent during pregnancy remain controversial.

- Lupus flares during pregnancy do not seem to be exceedingly more serious than those occurring in non-pregnant patients.

- Lupus may flare at any trimester and the postpartum period.

in their SLE patients involved the kidneys or the central nervous system as compared with $12 \%$ in the controls, but statistical significance could not be reached. ${ }^{13}$ Overall, the results from these studies do not suggest lupus flares during pregnancy are exceedingly more severe than those occurring outside pregnancy. However, given the high frequency of flares during pregnancy, SLE pregnancies should be regarded as high risk and close monitoring for disease activity is mandatory throughout the pregnancy course and the puerperium.

\section{Optimal timing for conception}

A number of studies have demonstrated that active lupus at the time of conception was associated with a higher risk of disease flares during pregnancy. ${ }^{15-18}$ The incidence of disease flares in patients with lupus nephritis undergoing pregnancies range from $7.4 \%$ to $63.0 \%{ }^{19}$ Renal flares during pregnancy may run an aggressive course with the development of acute renal failure and may even lead to maternal death. Houser et al studied 18 pregnancies from 11 patients with SLE who had nephritis and reported that $50 \%$ of patients with active disease at conception flared but for those with inactive disease, only $20 \%$ had flares. ${ }^{15}$ In another multicentre study by Hayslett et al that involved 65 pregnancies from 47 patients with history of lupus nephritis, it was described that of the 25 pregnancies with active renal disease at conception, $48 \%$ had flares of nephritis. ${ }^{16}$ This percentage was higher than that of pregnancies with inactive renal disease at the time of conception (32\%). Jungers et al reported that 12 of 26 (46\%) lupus pregnancies studied had disease flares. ${ }^{17}$ Those with active lupus nephritis at the time of conception had a higher chance of flares than those without (66\% v 9\%). Finally, Bobrie et al described the rate of disease flares during lupus pregnancies to be $62 \%$ and $7.4 \%$, respectively, for those with active and inactive nephritis at conception. ${ }^{18}$ Thus, in SLE patients, pregnancy is best undertaken during periods of quiescent disease and nephritis, if present, should be in remission for at least six months before conception. There are still no uniformly acceptable or standardised criteria for the definition of renal remission. As proposed by Boumpas and Balow, patients who have stabilisation of their renal function, resolution of urine sediment abnormalities, proteinuria of less than $1 \mathrm{~g}$ per day, and normalisation (ideally) of the C3 level for at least six months should be regarded as having renal remission. ${ }^{19}$ 
As a general rule, the longer the patient is in remission at the time of conception, the higher is the chance that she can complete the pregnancy without experiencing a disease exacerbation.

\section{Obstetric outcome of SLE}

Patients with SLE have an increased risk of pre-eclampsia during pregnancies. The incidence of pre-eclampsia in lupus pregnancies ranges from $5 \%$ to $38 \%$ in various reported series ${ }^{20}$ which is higher than that of pregnancies in women without SLE. Three recent retrospective studies have provided information regarding the course of pregnancy in patients with lupus nephritis. ${ }^{21-23}$ Hypertension developed frequently $(37 \%-56 \%)$ during pregnancies in these patients. Pre-eclampsia may develop in up to $30 \%$ of SLE patients with preexisting nephritis. ${ }^{23}$ Apart from the general risk factors such as primigravida, pre-existing systolic hypertension, smoking, obesity and previous history of pre-eclampsia, miscarriages, or abortions, ${ }^{24}$ the presence of aPL is an additional factor for early onset preeclampsia. ${ }^{25}$

Differentiation between pre-eclampsia and nephritic flare during pregnancy can be difficult. Both conditions can cause hypertension, proteinuria, oedema and worsening renal function, and may coexist in the same patient. Distinction between relapse of nephritis and pre-eclampsia is important because the management is different. There are some clinical clues that may help. First, as shown by Buyon et al, the serum C3 and C4 levels normally rise steadily during pregnancy and in patients with pre-eclampsia. ${ }^{26} \mathrm{~A}$ drop in C3 and $\mathrm{C} 4$, coupled with a rising anti-dsDNA titre, is likely to be associated with disease flare in a SLE patient with proteinuria. Second, the presence of active urinary sediments (white cell, red cell, or granular casts) and disease activity in other organs such as true arthritis, cutaneous vasculitis, oral ulcers, and lymphadenopathy in a patient with worsening proteinuria points to a lupus flare. Third, prednisone treatment will typically worsen pre-eclampsia while renal flare will respond to increasing dose of prednisone. Finally, according to the experience of the John Hopkins' SLE cohort, Petri mentioned that hypertension was not present in most of their patients with relapse of nephritis while raised blood pressure occurred almost universally in patients with pre-eclampsia. ${ }^{27}$

\section{Fetal outcome of SLE}

Pregnancies in SLE patients are characterised by an increased incidence of fetal wastage (abortions and stillbirths), prematurity, and intrauterine growth retardation (IUGR). ${ }^{20}$ The rates of abortion and stillbirths in lupus pregnancies vary from $6 \%-35 \%$ and $0 \%-22 \%$, respectively, which are higher than that of the general population. ${ }^{928}$ Active lupus nephritis, previous history of fetal death, and the presence of the aPL have been shown to be predictive factors for fetal wastages in lupus pregnancies. ${ }^{15-17}$ 29-33 In a recent study by Rahman et al active renal disease was found to
Box 2: Obstetric and fetal outcome in lupus pregnancies

- The incidence of pre-eclampsia is increased.

- Pre-existing hypertension, nephritis, and the presence of aPL are risk factors for pre-eclampsia.

- Fetal wastage, prematurity, and intrauterine growth retardation are more common.

- Active nephritis at conception and the presence of aPL predict for fetal losses.

be a significant predictor for fetal loss $(p<0.012) .^{34}$ Maternal hypertension was also identified as a significant statistical predictor for prematurity and IUGR. On the other hand, numerous prospective studies conducted in the 1990s have shown that in lupus pregnancies, mothers positive for aPL had a much higher risk of fetal loss than those who were negative. ${ }^{30}{ }^{33}$ In fact, recurrent pregnancy loss is a major clinical criterion for the antiphospholipid syndrome. Fetal loss related to the antiphospholipid syndrome usually occurs in the second and third trimesters. ${ }^{32}$ The presence of both the lupus anticoagulant and high titre IgG anticardiolipin antibodies (aCL) are associated with the highest risk of fetal wastage. ${ }^{33} 35$

Uncontrolled trials in the 1980s appeared to suggest that low dose aspirin (75-100 mg/day) and prednisone might improve fetal outcome in recurrent pregnancy loss associated with aPL. ${ }^{36}$ In 1992, Cowchock et al showed in a randomised controlled trial that maternal treatment with subcutaneous heparin (20000 $\mathrm{U} /$ day) and low dose aspirin $(80 \mathrm{mg} /$ day $)$ was as effective as prednisone ( $40 \mathrm{mg} /$ day) and low dose aspirin in terms of fetal outcome. ${ }^{37}$ As mothers treated with prednisone suffered from more morbidity such as pre-eclampsia, premature rupture of membranes and preterm deliveries, heparin was the preferred treatment to prednisone. Silver et al compared maternal morbidity and perinatal outcome in pregnant mothers with positive aPL who were treated with either aspirin $(81 \mathrm{mg} /$ day) alone or with combined therapy of aspirin and prednisone $(\geqslant 20 \mathrm{mg} /$ day) and found that preterm deliveries were more common in the prednisone treated group. ${ }^{38}$ However, fetal wastage was $0 \%$ in both arms. The surprisingly high successful pregnancy rate in this study might probably be related to the fact that their patients did not meet the strictest definition of antiphospholipid syndrome and had not had three consecutive pregnancy losses. Kutteh demonstrated that subcutaneous heparin in addition to aspirin $(81 \mathrm{mg} /$ day $)$ provided a significantly better pregnancy outcome than aspirin alone in recurrent pregnancy loss associated with aPL. ${ }^{39}$ The same author also described a similar efficacy between lower dose (average maximum dose $16254 \mathrm{U} /$ day) and higher dose (average maximum dose $26600 \mathrm{U} /$ day) of heparin in combination of low dose aspirin in the prevention of fetal loss in these patients. ${ }^{40}$ 
As a higher dose of heparin may be associated with more bleeding complications, most physicians prefer a dosage ranging from 5000 to 7500 units administered twice daily. Finally, a more recent study by Rai et al confirmed previous results that aspirin ( $75 \mathrm{mg} /$ day) combined with subcutaneous unfractionated heparin (10 $000 \mathrm{U} /$ day) was significantly more efficacious than aspirin alone in achieving live births in women with recurrent miscarriages associated with aPL. ${ }^{41}$

For primiparous mothers or multiparous SLE patients without a history of fetal loss but who have low titre IgG or IgM aCL, no specific therapy is usually recommended. Low dose aspirin should be considered for those patients who have high titre IgG aCL or positive lupus anticoagulant. High risk patients such as those with history of recurrent fetal losses should be treated with a combination of low dose aspirin and subcutaneous heparin. Although not formally studied in pregnancy, low molecular weight heparin seems to be a good alternative to unfractionated heparin in the treatment of antiphospholipid syndrome related pregnancy loss. ${ }^{36}$

\section{Neonatal lupus erythematosus (NLE) syndrome}

NLE is a syndrome consisting of congenital heart block (CHB), transient cutaneous lupus lesions, cytopenia, hepatic, and other systemic manifestations in children born to mothers with SLE, Sjogren's syndrome, or other rheumatic diseases with a positive anti-Ro or anti-La antibodies. The Research Registry for Neonatal Lupus in the States defines NLE by two criteria: (1) maternal antibodies to the 52 $\mathrm{kD}$ SSA/Ro, $60 \mathrm{kD} \mathrm{SSA} / \mathrm{Ro}$, or $48 \mathrm{kD} \mathrm{SSB} / \mathrm{La}$ ribonucleoproteins and (2) heart block or transient skin rash. ${ }^{42}$ The antibodies are usually cleared over weeks and most of the manifestations are mild and transient except for congenital heart block, which is permanent and carries significant mortality and morbidity to the offspring. ${ }^{43}$

$\mathrm{CHB}$ is the most common manifestation of the NLE syndrome. The estimated incidence of $\mathrm{CHB}$ in the general population is around one in 20000 live births $(0.005 \%) .^{44}$ Having SLE per se is not an independent risk factor for the development of CHB but rather depends solely on the presence of anti-SSA/Ro or antiSSB/La antibodies. For SLE patients with a positive anti-Ro, the risk of CHB is between $1.5 \%$ and $20.5 \%$, with an average figure of $7.2 \%$ after pooling the data from various studies. ${ }^{45}$ This is much higher than SLE mothers without the anti-Ro antibodies in whom the risk was estimated to be only $0.6 \%{ }^{46} \mathrm{~A}$ recently published report on the largest series of autoimmune CHB to date found an equal distribution between boys and girls. ${ }^{42}$ Julkunen et al studied 46 mothers with at least one child with $\mathrm{CHB}$ and controls with normal children and showed that the highest risk of CHB was observed in patients with positive anti-52 kD SSA/Ro by immunoblot (odds ratio 18.9; $95 \%$ confidence interval (CI) 7.7 to 46.5 , $\mathrm{p}<0.0001) .{ }^{44}$ The relative risk for a female child

\section{Box 3: Congenital heart block}

- Having SLE per se is not an independent risk factor but rather depends solely on the presence of anti-SSA/Ro or anti-SSB/La.

- The risk is approximately $7 \%$ in SLE mothers with positive anti-SSA/Ro.

compared to a male child to have CHB was 1.9 ( $95 \%$ CI 1.2 to $2.9, \mathrm{p}=0.09$ ). The risk of having another child with CHB in those who have already had one child with CHB is $12 \%$ (four of 34). This figure is similar to that reported by Buyon et al in which the recurrence rate of CHB in 49 mothers with subsequent pregnancies was $16 \%$ over a 27 year period. ${ }^{42}$

The pathogenesis of fetal CHB in mothers with anti-Ro or anti-La positivity is not completely understood. Circumstantial evidence has shown that the placental transfer of these antibodies occurs during the second trimester and mediates immunological injuries to the fetal heart and the conduction system. Attempts to prevent the placental transfer of harmful pathogenetic antibodies such as administration of corticosteroids, intravenous gammaglobulins, plasmapheresis, or in combination during the second trimester have been unsuccessful. ${ }^{47}$ Once complete heart block is established, it is irreversible.

Although anti-extractable nuclear antigen antibodies are not essential for routine disease monitoring in patients with SLE, screening for anti-Ro and anti-La antibodies is recommended for SLE patients who plan to be pregnant. Mothers with positive anti-Ro (especially the anti-52kD SSA/Ro) or anti-La antibodies are at risk of the NLE syndrome. Fetal echocardiography should be done during the 16 th to 24 th week of gestation by a specialist experienced in dealing with high risk mothers. In addition to an accurate diagnosis of CHB in utero, fetal echocardiography is also useful in following the course of the disease and detecting fetal myocarditis, pericardial effusion, and valvular regurgitation. When the clinical condition of the fetus deteriorates on serial echocardiograms (for example, development of heart failure and hydrops), maternal dexamethasone should be considered.

Safety of medications in lupus pregnancy There has been extensive literature regarding the safety of aspirin use during pregnancy. Exposure to big doses of aspirin ( $\geqslant 3 \mathrm{~g} /$ day) is associated with prolonged gestation and labour as well as increased bleeding complications during delivery. ${ }^{48}$ High doses of salicylates have also been associated with oligohydramnios, premature closure of the ductus arteriosus, and pulmonary hypertension. ${ }^{49}$ However, the incidence of fetal congenital abnormalities is not increased. ${ }^{5051}$ On the other hand, there is still no evidence that the non-steroidal antiinflammatory drugs (NSAIDs) currently available are teratogenic in human beings. However, high dose aspirin or NSAIDs should be 
avoided during the last few weeks of pregnancy because of their possible effects on uterine contraction, platelet function, and physiological changes that are taking place at and before birth in the fetus such as closure of the ductus arteriosus.

Although there are reports that corticosteroid use in pregnancy is associated with cleft palates in rabbits and mice, ${ }^{52} 53$ the occurrence of such congenital anomalies is rare in humans and there is no solid evidence that these are more common than the background incidence of congenital anomalies in normal pregnancies. Prednisone, prednisolone, and methylprednisolone have minimal placental transfer and are the drugs of choice during pregnancy. Fluorinated corticosteroids such as dexamethasone and betamethasone easily cross the placenta and should not be used unless there is intent to treat the fetus. So far, no major adverse effects of corticosteroids on babies have been reported in various published series of lupus pregnancies. ${ }^{11} 1318$ However, it should be noted that the use of high dose corticosteroids during pregnancy is associated with premature rupture of the membranes, IUGR, and precipitation of maternal complications such as gestational diabetes, hypertension, osteoporosis, and avascular bone necrosis. ${ }^{37} 38$ 54-56

Hydroxychloroquine is the commonest antimalarial drug used in SLE. Unlike chloroquine in which congenital anomalies have been reported for its use in pregnancy, no reports of hydroxychloroquine induced congenital malformations have been described so far in the literature..$^{57-59}$ In a recent publication, 36 infants of 33 mothers had no apparent congenital anomalies attributable to hydroxychloroquine. ${ }^{60}$ On the contrary, as there is good evidence that withdrawal of hydroxychloroquine may lead to lupus flares, ${ }^{58} 5961$ this should not be stopped unnecessarily during pregnancy in patients with SLE.

Azathioprine and cyclophosphamide are the two most commonly used cytotoxic agents used in the treatment of SLE. Both are teratogenic in animals. Azathioprine has not been associated with congenital defects in humans; however, sporadic anomalies such as preaxial polydactyly and bilateral pes quinovarus have been reported. ${ }^{62}$ There are many reports of fetal IUGR, lower birth weights and prematurity in kidney transplant recipients who received azathioprine and/or prednisone during pregnancy. ${ }^{556364}$ Yet, most large scale studies in transplant recipients have demonstrated that azathioprine is fairly well tolerated during pregnancy. Successful pregnancies have been reported in SLE patients who were treated with azathioprine and corticosteroids. ${ }^{65}$ Although increases in the rates of birth defects, miscarriages, and stillbirths have not been established in association of azathioprine use, the number of reported cases with adequate follow up may not be sufficient to detect a small increase in these rates or to detect late occurring abnormalities. For patients in whom immunosuppression is absolutely necessary during pregnancy (for example, those with
Box 4: Use of medications in lupus pregnancies

- High dose aspirin and NSAIDs should be avoided in the last few weeks of pregnancy.

- Corticosteroids and hydroxychloroquine have not been shown to be teratogenic.

- Azathioprine and cyclosporin A may be considered during pregnancy when intense immunosuppression is deemed necessary.

- Cyclophosphamide is teratogenic and should be avoided.

severe lupus), azathioprine is a reasonable choice and may be continued throughout pregnancy. Cyclophosphamide is teratogenic in humans. Although there are isolated case reports of successful pregnancies with cyclophosphamide and without congenital anomalies, long term follow up studies are scant and there is a risk of development of cancers in those exposed to cyclophosphamide in utero. ${ }^{66}$ Cyclophosphamide should therefore be avoided during pregnancy and appropriate contraception should be advised during periods of cyclophosphamide therapy.

Cyclosporin A is not an animal teratogen. Increasing data in human pregnancies suggest that there is no increased risk of congenital anomalies in exposed fetuses. In a large series of 115 renal transplant recipients with 154 pregnancies, cyclosporin A was found to be associated with lower birth weights but no malformations were observed. ${ }^{67}$ Another follow up study (mean 39 months) of 22 offsprings of mothers treated with cyclosporin A during pregnancy did not reveal that the drug is nephrotoxic to the exposed children. ${ }^{68}$ Although the long term effects of cyclosporin A exposure in utero are unknown, it may be considered as an alternative to other cytotoxic agents in the setting of severe disease activity in pregnant lupus patients.

\section{Lactation}

Most drugs used for the treatment of SLE are excreted into breast milk. After a single dose of aspirin ( $450-650 \mathrm{mg}$ ), $0.1 \%$ to $21 \%$ reaches the infant over a period of 24 hours. ${ }^{69}$ Peak salicylate concentration in milk occurs approximately two hours after the peak serum level. With immature neonatal metabolism, salicylate intoxication can theoretically occur in infants whose mothers are chronically taking antiinflammatory doses of aspirin. For this reason, the American Academy of Pediatrics (AAP) recommends that aspirin should be used with caution during breast feeding and big doses should be avoided. ${ }^{70}$

Most NSAIDs do not achieve high concentration in breast milk. The AAP considers ibuprofen, indomethacin, and naproxen to be safe with breast feeding. NSAIDs that exhibit enterohepatic circulation (for example, sulindac) are better avoided. Moreover, as NSAIDs displace bilirubin, they are contraindicated in 


\section{Box 5: Lactation}

- Big doses of aspirin should be avoided in nursing mothers.

- NSAIDs are contraindicated in nursing mothers with jaundiced neonates.

- Prednisone, prednisolone, and hydroxychloroquine are compatible with breast feeding.

- Breast feeding should not be contemplated in mothers who are taking cytotoxic agents such as cyclophosphamide, azathioprine, cyclosporin A, and methotrexate.

jaundiced neonates because of the increased risk of kernicterus. Overall, NSAIDs should be used cautiously in nursing mothers and those with a short half life and inactive metabolites are preferred. Alternative analgesics such as paracetamol should be considered.

Small amounts of corticosteroids can be found in the breast milk of women taking these drugs but no adverse reactions have been reported. The AAP considers prednisone and prednisolone compatible with breast feeding. Some clinicians recommend that mothers should wait for at least four hours before nursing when the dose of prednisone exceeds 20 $\mathrm{mg} /$ day. ${ }^{71}$ There are still no data on the use of dexamethasone or betamethasone in lactating mothers.

Low concentrations of hydroxychloroquine can be found in breast milk. Although the AAP classifies this drug as compatible with breast feeding, it should be used cautiously because of its slow elimination rate and potential accumulation to toxic amounts in the infant.

Substantial amount of cyclophosphamide can be found in the breast milk of nursing mothers and this drug is contraindicated during lactation. Breast feeding should not be contemplated in patients who require cyclophosphamide for disease control in the postpartum period. Other cytotoxic and immunosuppressive agents such as azathioprine, cyclosporin A, and methotrexate are also excreted in breast milk. The use of these agents is not recommended in nursing mothers because of the potential risks of immunosuppression, growth retardation, and carcinogenesis in the neonates.

\section{Breast feeding and disease activity of SLE}

Prolactin has multiple effects on the immune system and many of these are immunostimulatory. ${ }^{72}$ Prolactin concentrations normally rise in pregnancy during the second and third trimester. Hyperprolactinaemia persists for several months in patients who breast feed their children. Some earlier and small studies demonstrated that increased prolactin level in non-pregnant SLE patients was associated with disease activity. ${ }^{73}{ }^{74}$ However, later and larger studies did not confirm this result. ${ }^{75-78}$ The increase in prolactin levels may contribute to postpartum SLE flares, although the change in the concentrations of other hormones such as oestrogens and progestogens may also be responsible. A recent prospective study reported that first time breast feeders had increase activity of rheumatoid arthritis during the first six months after delivery when compared with those with rheumatoid arthritis but who did not breast feed. ${ }^{79}$ It was hypothesised that exacerbation of rheumatoid activity by breast feeding might be mediated by hormonal changes that included hyperprolactinaemia and was more likely to occur in genetically susceptible individuals. There are still no data regarding disease flare and breast feeding in SLE. The authors have reported a patient who had a postpartum relapse of SLE after a period of quiescent disease for more than four years. ${ }^{80}$ The disease flare was temporally related to breast feeding with documented hyperprolactinaemia. As the concentrations of other hormones were not assayed, it was difficult to be certain that hyperprolactinaemia per se had led to this flare and more cases and controls are needed to confirm this clinical observation.

\section{Contraception}

A number of anecdotal case reports have associated oestrogen-containing combined oral contraceptive pills with lupus exacerbation. ${ }^{81-84}$ This has led to reluctance of some physicians and gynaecologists in prescribing oral contraceptive pills to SLE patients. It should be noted that in most of these reports, a higher dose of ethinyl oestradiol ( $50 \mu \mathrm{g} / \mathrm{pill})$ was being used. As it is possible that the amount of oestrogen in the oral contraceptives may be related to the risk of lupus flares, the availability and trend of using a lower dose of ethinyl oestradiol (20-30 $\mu \mathrm{g} /$ day) in oral contraceptives is likely to reduce this risk.

While case reports of flares in SLE patients after oral contraceptive use might be coincidental, three retrospective studies, two with controls, have reached different conclusions regarding the association of oral contraceptives with SLE disease activity. Jungers et al reported a high flare up rate of $43 \%$ in a group of SLE patients with renal disease who received oral contraceptives. ${ }^{85}$ In another controlled study, Julkunen et al did not find an increase in the rate of flares during 12 months' follow up in 31 oral contraceptive users. ${ }^{86}$ Finally, Buyon et al found a very low self reported frequency of flare (seven of 55, 13\%) in SLE patients using oral contraceptives. ${ }^{87}$

In the absence of convincing controlled studies, it is still unclear whether oral contraceptive pills will exacerbate lupus. The ongoing Safety of Estrogens Lupus Erythematosus National Assessment (SELENA) trial conducted in the United States may hopefully provide more information on this aspect. ${ }^{88}$ As there is some evidence that the risk of thromboembolism related to contraceptive pill use may be higher in SLE patients, particularly in those with positive aPL, ${ }^{89}{ }^{90}$ oral contraceptives should be advised against in this subset of patients. For young ( $\leqslant 35$ years of age), non-smoking, and normotensive SLE patients 


\section{Box 6: Contraception in SLE patients}

- Low dose oestrogen-containing contraceptive pills may be considered for those patients with stable disease and without history of thromboembolism or aPL.

- Barrier methods and progestogens are alternatives if oestrogens are contraindicated.

- Intrauterine contraceptive device is associated with an increased risk of infection.

without aPL or other thrombotic risk factors such as family or personal history of thrombosis, and with stable disease for a considerable period of time, oral contraceptives containing low dose synthetic oestrogens should not be contraindicated. Close monitoring for disease activity and signs of thromboembolism is mandatory during the period of oral contraceptive use.

For patients who are not candidates for oestrogen-containing oral contraceptives, progestogens may be an alternative form of hormonal contraception. However, progestogen only pills and depot progestogens (for example, Depo-Provera) are associated with a number of side effects such as menstrual irregularities, spotting, amenorrhoea, oedema, and change in body weight.

Intrauterine contraceptive devices are associated with increase incidence of infection, particularly in those SLE patients who are under heavy immunosuppression and is therefore not usually recommended. Mechanical barrier methods such as condoms with spermicides and diaphragms are safe and effective and may be suitable for those patients who are contraindicated for contraceptive methods described above.

\section{Uraemia and pregnancy risk}

Occasionally patients with inactive lupus, but with different degrees of renal impairment caused by previous episodes of nephritis, may come for advice on the risks of pregnancy related to chronic renal disease in general. A study of 25 pregnancies from 23 women with moderate renal insufficiency showed that a decline in renal function was fairly common during pregnancy and might be more rapid than that expected from the natural history of the disease. ${ }^{91}$ The functional deterioration ranged from a mild increase in serum creatinine concentration to a state that required dialysis. Hypertension developed or worsened in more than half of these pregnancies and premature delivery was usual. Chronic renal failure is often associated with amenorrhoea, anovulatory cycles, and reduced libido and the chance of conception is low. ${ }^{92}$ While successful pregnancies are definitely possible in uraemic patients, the maternal and fetal risks during pregnancy should be minimised by aggressive control of blood pressure and prompt diagnosis and treatment of bleeding episodes. Patients on dialysis should be given adequate dialysis to maintain serum biochemistries to as normal as possible. Premature labour should be treated with NSAIDs. Couples should be thoroughly counselled about the serious risks of pregnancy in uraemic patients and close collaboration with nephrologists is necessary.

\section{Conclusion}

Patients with SLE have normal fertility and should not be discouraged from having children. A thorough and detailed discussion with couples regarding the risk of disease flares, optimal timing of conception, possible maternal and fetal complications during pregnancy and their consequences is essential in relieving anxiety from the patient and ensuring a satisfactory pregnancy outcome. Pregnancy is best undertaken when the general health of the patient is at its best and when the disease, especially lupus nephritis, is in clinical remission for at least six months. Appropriate counselling for the risk of neonatal lupus syndrome should also be given to those patients with positive anti-Ro or anti-La antibodies. Aspirin and/or subcutaneous heparin should be considered for those who have recurrent miscarriages and positive antiphospholipid antibodies. Pregnancy in women with SLE is a high risk and judicious monitoring for disease flares and thromboembolic phenomena during the pregnancy course and the puerperial period is mandatory. Blood pressure, urine protein, creatinine clearance, complement concentrations, antidsDNA titres, and blood counts should be obtained at each antenatal visit and close collaboration with obstetricians and perinatologists is essential. Regular surveillance for the well being of the fetus can be achieved by methods such as the non-stress test and biophysical profile. Fetal ultrasonography and echocardiography is useful in picking up CHB during the second trimester of pregnancy and monitoring for progress.

1 Khamashta MA, Hughes GRV. Pregnancy in systemic lupus erythematosus. Curr Opin Rheumatol 1996;8:424-9.

2 Kaufman RL, Kitridou RC. Pregnancy in mixed connective tissue disease: comparison with systemic lupus erythematosus. F Rheumatol 1982;9:549-55.

3 Nossent HC, Swaak TJ. Systemic lupus erythematosus. VI. Analysis of the interrelationship with pregnancy. $\mathcal{F}$ Rheumatol 1990;17:771-6.

4 Buyon JP, Wallace DJ. The endocrine system, use of exogenous estrogens, and the urogenital tract. In: Wallace DJ, Hahn BH, eds. Dubois' lupus erythematosus. 5th Ed. Baltimore: William \& Wilkins, 1997: 817-34.

5 Langevitz P, Klein L, Pras M, et al. The effect of cyclophosphamide pulses on fertility in patients with lupus nephritis. Am $\mathcal{F}$ Reprod Immunol 1992;28:157-8.

6 Mok CC, Lau CS, Wong RWS. Risk factors for ovarian failMok CC, Lau CS, Wong RWS. Risk factors for ovarian fail-
ure in patients with systemic lupus erythematosus receiving ure in patients with systemic lupus erythematosus receiving
cyclophosphamide therapy. Arthritis Rheum 1998;41:831-7. 7 Boumpas DT, Austin HA, Vaughan EM, et al. Risk of sustained amenorrhoea in patients with systemic lupus erythematosus receiving intermittent pulse cyclophosphamide therapy. Ann Intern Med 1993;119:366-9.

8 Lockshin MD, Reinitz E, Druzin ML, et al. Lupus pregnancy. Case-control prospective study demonstrating absence of lupus exacerbation during or after pregnancy. Am F Med 1984;77:893-8.

9 Mintz G, Niz J, Gutierrez G, et al. Prospective study of pregnancy in systemic lupus erythematosus. Results of a multidisciplinary approach. F Rheumatol 1986;13:732-9.

10 Urowitz MB, Gladman DD, Farewell VT, et al. Lupus and pregnancy studies. Arthritis Rheum 1993;36:1392-7.

11 Wong KL, Chan FY, Lee CP. Outcome of pregnancy in Wong KL, Chan FY, Lee CP. Outcome of pregnancy in
patients with systemic lupus erythematosus. A prospective patients with systemic lupus erythematos
study. Arch Intern Med 1991;151:269-73.

12 Petri M, Howard D, Repke J. Frequency of lupus flare in pregnancy. The Hopkins Lupus Pregnancy Center experi-
ence. Arthritis Rheum 1991;34:1538-45. 
13 Ruiz-Irastorza G, Lima F, Alves J, et al. Increased rate of lupus flare during pregnancy and the puerperium: a
prospective study of 78 pregnancies. $B r \quad \mathcal{F}$ Rheumatol prospective study

14 Buyon JP, Kalunian KC, Ramsey-Goldman R, et al. Assessing disease activity in SLE patients during pregnancy. Lupus 1999;8:677-84.

15 Houser MT, Fish AJ, Tagatz GE, et al. Pregnancy and systemic lupus erythematosus. Am F Obstet Gynecol 1980; 138:409-13

16 Hayslett JP, Lynn RI. Effect of pregnancy in patients with lupus nephropathy. Kidney Int 1980;18:207-20.

17 Jungers P, Dougados M, Pelissier C, et al. Lupus nephropathy and pregnancy. Report of 104 cases in 36 patients. Arch thy and pregnancy. Report of
Intern Med 1982;142:771-6.

18 Bobrie G, Liote F, Houillier P, et al. Pregnancy in lupus nephritis and related disorders. Am F Kidney Dis 1987;9: nephritis

19 Boumpas DT, Balow JE. Outcome criteria for lupus nephritis trials: a critical overview. Lupus 1998;7:622-9.

20 Kitridou RC. The mother in systemic lupus erythematosus. In: Wallace DJ, Hahn BH, eds. Dubois' lupus erythematosus. 5th Ed. Baltimore: Williams \& Wilkins, 1997: 967-1002.

21 Oviasu E, Hicks J, Cameron JS. The outcome of pregnancy in women with lupus nephritis. Lupus 1991;1:19-25.

22 Packham DK, Lam SS, Nicholls K, et al. Lupus nephritis and pregnancy. $Q 7 \mathrm{Med} 1992 ; 83: 315-24$.

23 Julkunen H, Kaaja R, Palosuo T, et al. Pregnancy in lupus nephropathy. Acta Obstet Gynecol Scand 1993;72:258-63.

24 Sibai BM, Gordon T, Thom E, et al. Risk factors for preeclampsia in healthy nulliparous women: a prospective multicenter study. The National Institute of Child Health and Human Development Network of Maternal-Fetal Medicine Human Development Network of Maternal-

25 Dekker GA, de Vries JI, Doelitzsch PM, et al. Underlying disorders associated with severe early-onset preeclampsia. Am f Obstet Gynecol 1995;173:1042-8.

26 Buyon JP, Cronstein BN, Morris M, et al. Serum complement values (C3 and C4) to differentiate between systemic lupus activity and pre-eclampsia. Am f Med 1986; 21:194-200.

27 Petri M. Pregnancy in SLE. Ballieres Clin Rheumatol 1998;12:449-76.

28 Nossent HC, Swaak TJ. Systemic lupus erythematosus. VI. Analysis of the interrelationship with pregnancy. F Rheumatol 1990;17:771-6.

29 Martinez-Rueda JO, Arce-Salinas CA, Kraus A, et al. Factors associated with fetal losses in severe systemic lupus erythematosus. Lupus 1996;5:113-19.

30 Out HJ, Bruinse HW, Christiaens GC, et al. A prospective, controlled multicenter study on the obstetric risks of pregnant women with antiphospholipid antibodies. $A m$ f pregnant women with antiphosph
Obstet Gynecol 1992;167:26-32.

31 Loizou S, Byron MA, Englert HJ, et al. Association of quantitative anticardiolipin antibody levels with fetal loss and time of loss in systemic lupus erythematosus. $Q \mathcal{F}$ Med 1988; 68:525-31.

32 Ginsberg JS, Brill-Edwards P, Johnston M, et al. Relationship of antiphospholipid antibodies to pregnancy loss in patients with systemic lupus erythematosus: a crosssectional study. Blood 1992;80:975-80

33 Lockshin MD, Druzin ML, Goei S, et al. Antibody to cardiolipin as a predictor of fetal distress or death in pregnant patients with systemic lupus erythematosus. $N$ Engl f Med 1985;313:152-6.

34 Rahman P, Gladman DD, Urowitz MB. Clinical predictors of fetal outcome in systemic lupus erythematosus. $\mathcal{f}$ Rheumatol 1998;25:1526-30.

35 Ninomiya C, Taniguchi O, Kato T, et al. Distribution and clinical significance of lupus anticoagulant and anticardiolipin antibody in 349 patients with systemic lupus erythematosus. Intern Med 1992;31:194-9.

36 Welsch S, Branch DW. Antiphospholipid syndrome in pregnancy. Obstetric concerns and treatment. Rheum Dis Clin North Am 1997;23:71-84.

37 Cowchock FS, Reece EA, Balaban D, et al. Repeated fetal losses associated with antiphospholipid antibodies: a collaborative randomized trial comparing prednisone with lowdose heparin treatment. Am $\mathcal{f}$ Obstet Gynecol 1992;166: 1318-23.

38 Silver RK, MacGregor SN, Sholl JS, et al. Comparative trial of prednisone plus aspirin versus aspirin alone in the treatment of anticardiolipin antibody-positive obstetric patients. Am f Obstet Gynecol 1993;169:1411-17.

39 Kutteh WH. Antiphospholipid antibody-associated recurrent pregnancy loss: treatment with heparin and low-dose
aspirin is superior to low-dose aspirin alone. Am $\mathcal{F}$ Obstet aspirin is superior to low-
Gynecol 1996;174:1584-9.

40 Kutteh WH, Ermel LD. A clinical trial for the treatment of antiphospholipid antibody-associated recurrent pregnancy loss with lower dose heparin and aspirin. Am $\mathcal{F}$ Reprod Immunol 1996;35:402-7.

41 Rai R, Cohen H, Dave M, et al. Randomised controlled trial of aspirin and aspirin plus heparin in pregnant women with recurrent miscarriage associated with antiphospholipid antibodies. BMF 1997;314:253-7.

42 Buyon JP, Hiebert R, Copel J, et al. Autoimmune-associated congenital heart block: demographics, mortality, morbidity and recurrence rates obtained from a national neonatal lupus registry. $7 \mathrm{Am}$ Coll Cardiol 1998;31:1658-66.

43 Meng C, Lockshin M. Pregnancy in lupus. Curr Opin Rheumatol 1999;11:348-51.

44 Julkunen H, Kaaja R, Siren MK, et al. Immune-mediated congenital heart block (CHB): identifying and counseling patients at risk for having children with CHB. Semin Arthritis Rheum 1998;28:97-106.

45 Kitridou RC. The fetus in systemic lupus erythematosus. In: Wallace DJ, Hahn BH, eds. Dubois'lupus erythematosus. 5th Ed. Baltimore: Williams \& Wilkins, 1997: 1003-21.

46 Ramsey-Goldman R, Hom D, Deng JS, et al. Anti-SS-A antibodies and fetal outcome in maternal systemic lupus erythematosus. Arthritis Rheum 1986;29:1269-73.

47 Tseng CE, Buyon JP. Neonatal lupus syndromes. Rheum Dis Clin North Am 1997;23:31-54.

48 Lewis RB, Schulman JD. Influence of acetylsalicylic acid, an inhibitor of prostaglandin synthesis, on the duration of human gestation and labour. Lancet 1973;ii:1159-61.

49 Lockshin MD, Sammaritano LR, Schwartzman S. Lupus pregnancy. In: Lahita RG, ed. Systemic lupus erythematosus. 3rd Ed. New York: Academic Press, 1999: 507-36.

50 Turner G, Collins E. Fetal effects of regular salicylate ingestion in pregnancy. Lancet 1975;ii:338-9.

51 Jick H, Holmes LB, Hunter JR, et al. First-trimester drug Jick $\mathrm{H}$, Holmes LB, Hunter JR, et al. First-trimester
use and congenital disorders. $\mathscr{F} A M A$ 1981;246:343-6.

52 Fraser FC, Fainstat TD. Production of congenital defects in the offspring of pregnant mice treated with cortisone. Pediatrics $1951 ; 8: 527-30$

53 Pinsky L, DiGeorge AM. Cleft palate in the mouse: a teratogenic index of glucocorticoid potency. Science 1965;147: $402-5$.

54 Khamashta MA, Ruiz-Irastorza G, Hughes GRV. Systemic lupus erythematosus flares during pregnancy. Rheum Dis Clin North Am 1997;23:15-29.

55 Bermas BL, Hill JA. Effects of immunosuppressive drugs during pregnancy. Arthritis Rheum 1995;38:1722-32.

56 Branch DW, Silver RM, Blackwell JL, et al. Outcome of treated pregnancies in women with antiphospholipid syndrome: an update of the Utah experience. Obstet Gynecol 1992;80:614-20.

57 Wallace DJ. Antimalarial agents and lupus. Rheum Dis Clin North Am 1994;20:243-63.

58 Parke AL, West B. Hydroxychloroquine in pregnant patients with systemic lupus erythematosus. I Rheumatol 1996;23: 1715-18.

59 Parke AL, Rothfield NF. Antimalarial drugs in pregnancythe North American experience. Lupus 1996;5(suppl):S67-9.

60 Buchanan NM, Toubi E, Khamashta MA, et al. Hydroxychloroquine and lupus pregnancy: review of a series of 36 cases. Ann Rheum Dis 1996;55:486-8.

61 The Canadian Hydroxychloroquine Study Group. A randomized study of the effect of withdrawing hydroxychloroquine sulfate in systemic lupus erythematosus. $N$ Engl $\mathcal{F}$ Med 1991;324:150-4.

62 Ramsey-Goldman R, Schilling E. Immunosuppressive drug use during pregnancy. Rheum Dis Clin North Am 1997;23: 149-67.

63 Armenti VT, Ahlswede KM, Ahlswede BA, et al. National Transplantation Pregnancy Registry: analysis of outcome/ risks of 394 pregnancies in kidney transplant recipients. Transplant Proc 1994;26:2535.

64 Pirson Y, Van Lierde M, Ghysen J, et al. Retardation of fetal growth in patients receiving immunosuppressive therapy. $N$ Engl F Med 1985;313:328

65 Ramsey-Goldman R, Mientus JM, Kutzer JE, et al Pregnancy outcome in women with systemic lupus erythematosus treated with immunosuppressive drugs. F Rheu-
matol 1993;20:1152-7.

66 Zemlickis D, Lishner M, Erlich R, et al. Teratogenicity and carcinogenicity in a twin exposed in utero to cyclophosphamide. Teratog Carcinog Mutagen 1993;13:139-43.

67 Armenti VT, Ahlswede KM, Ahlswede BA, et al. National transplantation Pregnancy Registry-outcomes of 154 pregnancies in cyclosporine-treated female kidney transpregnancies in cyclosporine-treated female kidn
plant recipients. Transplantation 1994;57:502-6.

68 Shaheen FAM, Al-Sulaiman MH, Al-Khader AA. Longterm nephrotoxicity after exposure to cyclosporin in utero. term nephrotoxicity after expos

69 Berlin CM, Pascuzzi MJ, Yaffe SJ. Excretion of salicylate in human milk. Clin Pharmacol Ther 1980;27:245-8.

0 Committee on Drugs, American Academy of Pediatrics. The transfer of drugs and other chemicals into human milk. Pediatrics 1994;93:137-50.

71 Ost L, Wettrell G, Bjorkhem I, et al. Prednisone excretion in human milk. F Pediatr 1985;106:1008-11.

72 Reber PM. Prolactin and immunomodulation. Am 7 Med 1993;295:637-44.

73 Jara-Quezada L, Graef A, Lavalle C. Prolactin and gonadal hormones during pregnancy in systemic lupus erythematosus. F Rheumatol 1991;18:349-53.

74 McMurray RW, Allen SH, Braun AL, et al. Longstanding hyperprolactinemia associated with systemic lupus erythematosus: possible hormonal stimulation of an autoimmune disease. f Rheumatol 1994;21:843-50.

75 Pauzner R, Urowitz MR, Gladman DD, et al. Prolactin in systemic lupus erythematosus. F Rheumatol 1994;11:2064-7.

76 Buskila D, Lorber M, Neumann L, et al. No correlation between prolactin levels and clinical activity in patients with systemic lupus erythematosus. F Rheumatol 1996;23:62932 .

77 Ostendorf B, Fischer R, Santen R, et al. Hyperprolactinemia in systemic lupus erythematosus? Scand 7 Rheumatol 1996;25:97-102.

78 Mok CC, Lau CS, Tam SCF. Prolactin profile in a cohort of Chinese systemic lupus erythematosus patients. $\mathrm{Br} \quad \mathrm{f}$ Rheumatol 1997;36:986-9.

79 Barrett JH, Brennan P, Fiddler M, et al. Breast-feeding and postpartum relapse in women with rheumatoid and inflammatory arthritis. Arthritis Rheum 2000;43:1010-15. 
80 Mok CC, Wong RWS, Lau CS. Systemic lupus erythemato-

81 Chapel TA, Burns RE. Oral contraceptives and exacerbation of lupus erythematosus. Am f Obstet Gynecol 1971;110: tion of

82 Pimstone BL. Systemic lupus erythematosus exacerbated by oral contraceptives. S Afr f Obstet Gynecol 1966;4:62-3.

83 Furakawa F, Tachibana T, Imamura S, et al. Oral contraceptive-induced lupus erythematosus in a Japanese woman. F Dermatol 1991;18:56-8.

84 Garovich M, Agudelo C, Pisko E. Oral contraceptives and systemic lupus erythematosus. Arthritis Rheum 1980;23: 1396-8.

85 Jungers $\mathrm{P}$, Dougados M, Pelissier C, et al. Influence of oral contraceptive therapy on activity of systemic lupus erythematosus. Arthritis Rheum 1982;25:618-23.

86 Julkunen HA. Oral contraceptives in systemic lupus erythematosus: side effects and influence on the activity of erythematosus: side effects and influence
SLE. Scand $\mathcal{F}$ Rheumatol 1991;20:427-33.
87 Buyon JP, Kalunian KC, Skovron ML, et al. Can women with systemic lupus erythematosus safely use exogenous estrogens? F Clin Rheumatol 1995;1:205-12.

88 Petri M, Buyon J, for the SELENA Group. Flares in the SELENA oral contraceptive trial [abstract]. Arthritis Rheum 1999;42:S265.

89 Julkunen HA, Kaaja R, Friman C. Contraceptive practice in women with systemic lupus erythematosus. $\mathrm{Br} \mathcal{F}$ Rheumatol 1993;32:227-30.

90 Asherson RA, Harris EN, Hughes GR, et al. Complications of oral contraceptives and antiphospholipid antibodies [letter]. Arthritis Rheum 1988;31:575-6.

91 Hou SH, Grossman SD, Madias NE. Pregnancy in women with renal disease and moderate renal insufficiency. $A m$ f Med 1985;78:185-94.

92 Hou SH. Pregnancy in women on hemodialysis and peritoneal dialysis. Baillieres Clin Obstet Gynecol 1994;8:481-500. 